\title{
Serial anticipation pattern learning in two-element and three-element series
}

\author{
E. J. CAPALDI, ROBERT D. BLITZER, and PATRICIA MOLINA \\ Purdue University, West Lafayette, Indiana 47907
}

\begin{abstract}
Serial anticipation learning under monotonically decreasing serial patterns was examined in relation to associative-chaining and cognitive approaches. The designation 8-0 describes a two-element serial pattern employing eight pellets on Trial 1 of each day and zero pellets on Trial 2. This investigation examined three two-element serial patterns $(8-0,4-0,2-0)$ and six three-element serial patterns (16-2-0, 8-2-0, 4-2-0, 2-2-0, 16-8-0, 16-8-0, 16-4-0). Better anticipation of the zero-pellet element occurred under the two-element patterns. Among three-element patterns, 16-2-0 and 8-2-0 produced the best anticipation. Serial anticipation learning was sometimes better earlier in training than later in training. Where two- and three-element groups received the same reward magnitude on Trial 1 of each day (e.g., 8-0 vs. 8-2-0), slower Trial 1 running was associated with the two-element pattern.
\end{abstract}

Serial order behavior in general and serial anticipation pattern learning in particular may provide an important link between animal and human learning and memory. Theories of serial anticipation pattern learning in the human area are generally cognitively oriented and stress rule learning (e.g., this pattern is a nonmonotonically decreasing one). It has been suggested that the rule approach may be appropriate in the animal area (e.g., Hulse, 1978; Hulse \& Dorsky, 1977). Many workers in the human area reject associative-chaining hypotheses or hypotheses that suggest that one element of a series gives rise to stimuli that control responding to the next element of the series (see, e.g., Crowder, 1976). Other human workers, however, have suggested that chaining hypotheses may be appropriate or not, depending upon the kind of serial order behavior being studied (see, e.g., Slamecka, 1977; Wickelgren, 1969).

Restle and Brown (1970) have made the valid and important point that serial anticipation learning demands the assumption that the anticipation of a current event may be based on several prior events. The point is as applicable to rat as to human serial anticipation learning and, quite importantly, it does not rule out chaining hypotheses, demanding only that they be more sophisticated than heretofore. Consider, for example, the sequential hypothesis (e.g., Capaldi, 1967), which is a chaining hypothesis and the only animal learning theory to directly concern itself with serial anticipation learning. According to this view, the memory produced by a given event, for example, nonreward, may be progressively modified over successive nonrewarded trials. This stimulus modification assumption has been employed to explain a wide variety of partial reward and delay of reward extinction findings (Capaldi, 1967),

This research was supported in part by National Institute of Child Health and Development Grant HD 04379 to E. J. Capaldi. as well as to explain serial anticipation learning in which running became progressively more rapid over a $4 \mathrm{NR}$ schedule, one in which four nonrewarded trials always preceded a rewarded trial (Capaldi, 1979). Thus running speed following a nonrewarded trial differed depending upon the number of prior nonrewarded trials: zero, one, two, or three. Restle and Brown (1970) have defined branching as follows: Anticipation following a given event differs depending upon prior events. Clearly, progressively increasing speed over the trials of a 4NR schedule is an example of branching and one predictable stressing that memory is modified over successive nonrewarded trials. Such stimulus modification, then, explains "conditional responding" or branching and, in a chaining context, exercises some of the functions of rules in a cognitive context.

It may be that associative and cognitive approaches are complementary as well as competitive; data exist in support of each approach (Capaldi \& Molina, in press; Hulse \& Dorsky, 1977). The central concern of this investigation was to determine if serial patterns considered easiest to anticipate from a sequential chaining viewpoint would in fact prove to be so. To this end, three two-element serial patterns and six threeelement serial patterns were examined.

\section{METHOD}

\section{Subjects}

The subjects, 72 male Sprague-Dawley rats obtained from the Holtzman Company, were about 100 days old at the start of the experiment. They were individually housed on a 16-h light/8-h dark cycle with free access to water.

\section{Apparatus}

A straight gray runway was used, measuring $203.5 \mathrm{~cm}$ long, $10.5 \mathrm{~cm}$ wide, and $22.4 \mathrm{~cm}$ high, and covered with $1.3-\mathrm{cm}$ hardware cloth. The alley was divided into three sections, start, run, and goal, by two unpainted brass guillotine doors. 
Raising the doors started the first of three .01-sec clocks. Interrupting a photobeam located $7.0 \mathrm{~cm}$ beyond the first door stopped the first clock (start time) and started the second clock. Interrupting a second photobeam located $131.4 \mathrm{~cm}$ beyond the first stopped the second clock (run time) and started the third clock. The interruption of a third photobeam, located $32.7 \mathrm{~cm}$ beyond the second photobeam and $6.5 \mathrm{~cm}$ in front of a brass $6.7 \times 10.5 \times 4.4 \mathrm{~cm}$ food cup, stopped the third clock (goal time). The food cup contained a brass inset measuring $3.8 \times 3.2 \times 1.6 \mathrm{~cm}$, which held food pellets on rewarded trials. The second guillotine door served to confine the rat to the goal section.

\section{Procedure}

Upon arrival at the laboratory, the animals were given free access to food. On Day 1 of the experiment, all food was removed from the home cages and, for the duration of the experiment, the rats were maintained on $13 \mathrm{~g}$ of food per day, including rewards received in the alley. On Days 1-14 they were briefly handled individually, and for the last 6 days of this period, the rats were given eight .045-g Noyes food pellets in the home cages.

Runway training began on Day 15 and continued for 24 days. Animals were randomly assigned to nine groups and received two or three trials per day, depending on group, for the duration of the experiment. The groups, designated by the number of pellets received on each trial, were as follows: 16-8-0, 16-4-0, 16-2-0, 8-2-0, 4-2-0, 2-2-0, 8-0, 4-0, 2-0. On nonrewarded trials, subjects were confined in the goalbox for $20 \mathrm{sec}$.

Rats were run in squads of nine composed of one animal from each group. The within-squad running order was the same for all squads and varied randomly each day, while the squads were run in the same order each day. The first trial for all three-trial subjects in a squad was run first, then the second trial for those subjects and the first trial for the two-trial subjects, and finally, the last (zero-pellet) trial for all animals. The rats were placed in holding cages between trials, where water was freely available. When the three-trial subjects were run on the first trial and a two-trial subject came up in the running order, the experimenter waited $1 \mathrm{~min}$ before running the next animal in order to maintain the intertrial interval at about $10 \mathrm{~min}$. Following the last trial of a squad, the animals were returned to the home cages and, at least 15 min later, fed their daily rations.

\section{RESULTS AND DISCUSSION}

Running speed $(1 / \mathrm{sec})$ to each element in blocks of 2 days was examined. Major aspects of the present findings may be conveyed by examining performance on Blocks 1, 5, 7, and 12. Such performance is shown in Figure 1 for the three-element groups (left panel) and the two-element groups (right panel). As can be seen, correct anticipation was not, as expected, evident on the first block of trials but had developed, more in some groups than in others, by the fifth block of trials. Attend first to the three-element groups. On the first block of trials, no difference of any consequence was significant; by Block 5 the groups differed significantly $[\mathrm{F}(5,42)=2.85, \mathrm{p}<.05]$, and the Trials by Groups interaction was significant as well $[F(10,84)=3.32$, $\mathrm{p}<.01]$. This pattern of findings, except for variation in significance level, was obtained on subsequent blocks.

Completely appropriate anticipation in a threeelement pattern would consist of faster running to Element 1 than to Element 2 and faster running to Element 2 than to Element 3. Post hoc analysis using

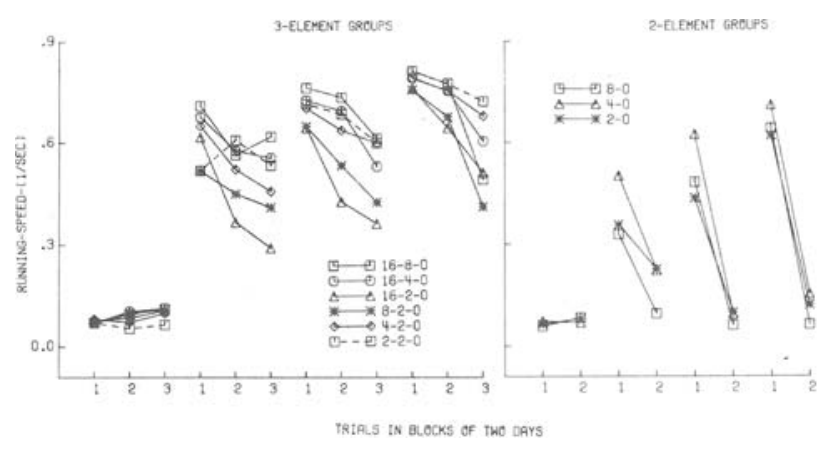

Figure 1. Running speed $(1 / \mathrm{sec})$ on each of the trials in the three-element groups (left panel) and the two-element groups on Blocks 1, 5, 7, and 12.

Newman-Keuls tests showed that only Group 16-2-0 showed such completely correct anticipation (all ps $<.05$ ) on a stable basis; it appeared by Block 8 (not depicted) and was present on Block 12. Group 8-2-0 showed complete anticipation on Block 7 but not again. It is not unusual for serial patterning to become maximal early in training and then to decrease (Capaldi, 1979). Thus, on Block 5 Group 4-2-0 ran faster on Trial 1 than on Trials 2 and 3 (ps $<.05$ ), but on subsequent blocks no patterning was evident. Again in Group 2-2-0 on Block 7, speed on Trial 1 was faster than speed on Trial 3 ( $p<.05$ ), with no significant patterning occurring on subsequent blocks. It is to be emphasized that an early appearance of correct anticipation and its eventual disappearance or reduction is, in many instances, not a chance event (see Capaldi, 1979). The two remaining three-element groups showed good anticipation: Their single difficulty was in not running slower on Trial 2 than on Trial 1. This is not too surprising with respect to Group 16-8-0 and perhaps is understandable in the case of Group 16-4-0, given the relatively large size of the Trial 2 rewards.

The two-element groups obviously anticipated the zero-pellet element. For example, on Block 7 the F for trials was 264.46 . The two-element groups obviously ran more slowly on zero-pellet trials than did the threeelement groups and differed little among themselves. Statistical findings completely support these statements. For example, on Block 12, group differences in running speed on the zero-pellet trial were highly significant $[F(8,63)=14.86, p<.001]$, and Newman-Keuls tests indicated that all two-element groups ran more slowly than all three-element groups (all ps $<.01$ or better).

Finally, there is an interesting and not completely expected finding. When two- and three-element groups receiving the same reward magnitude on Trial 1 are compared ( $2-0$ vs. $2-2-0,4-0$ vs. $4-2-0$, and $8-0$ vs. $8-2-0)$, it is clear that the two-element groups were slower on Trial 1. Figure 2 shows speed of running for these groups on Trial 1 of the day in blocks of 2 days. Interestingly, on the last two blocks of trials, while neither magnitude of reward $[F(2,42)=1.28, p>.05]$ nor the interaction 


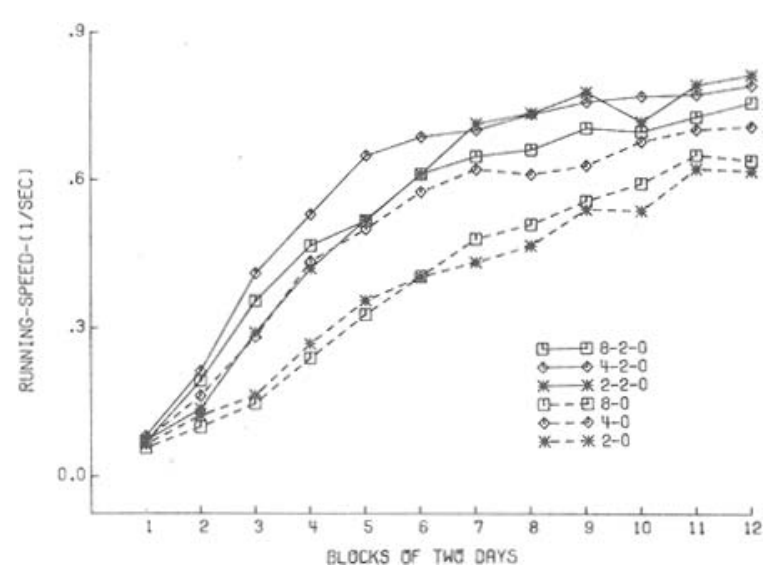

Figure 2. Running speed (1/sec) in 2-day blocks on Trial 1 of the day in the three-element groups, 2-2-0, 4-2-0, and 8-2-0 and in the two-element groups, 2-0, 4-0, and 8-0.

of magnitude and number of elements $[\mathrm{F}(2,42)=1.56$, $\mathrm{p}>.05$ ] produced significant differences, number of elements did $[F(1,42)=22.23, p<.001]$, indicating that number of elements affected Trial 1 running powerfully, more powerfully than reward magnitude itself.

Capaldi and Molina (in press) conceptualized serial anticipation learning as a discrimination problem using memories as discriminanda. Consider, for example, the 16-2-0 series. The memory produced by the 16-pellet reward, $S^{16}$, is retrieved on Trial 2 and so controls anticipatory running on that trial, while the memory produced by the two-pellet reward, $S^{2}$, is retrieved on Trial 3 and so controls anticipatory running to the zero-pellet element. As expected by the sequential view, anticipation was best in the 16-2-0 series because it provides a relatively easy discrimination considering three-element series. That is, the sequential view suggests that memories are arrayed along a stimulus continuum with large reward on the left and small reward on the right (see, e.g., Capaldi, 1967). Thus, $\mathrm{S}^{\mathbf{1}}$ is more similar to $S^{8}$ than to $S^{4}$, and so on. Among three-element groups, the easiest discrimination was between $S^{\mathbf{1 6}}$ and $S^{2}$, and this group did indeed anticipate every trial on a stable basis. Consider now two- vs. three-element groups.

A major and obvious difference between two- and three-element groups is that the three-element groups were rewarded on Trial 2 of each day. The simple assumption that some conditioning occurred on Trial 2 of the day in three-element groups that generalized to Trial 1 and Trial 3 cues explains a variety of findings. Clearly, it explains why three-element groups were faster on zero-pellet trials than two-element groups. By the same reasoning, it explains faster Trial 1 responding in three-element than in two-element groups receiving the same reward magnitude on Trial 1 . Finally, and this is more speculative, if it is assumed that in Groups 16-2-0, 8-2-0, 4-2-0, and 2-2-0 much of the Trial 2 conditioning occurred on the later trials, a basis is available for understanding why in these groups anticipation learning was better earlier than later in training (see, e.g., Capaldi, 1974).

A rule approach (Hulse, 1977; Hulse \& Dorsky, 1977) might explain differences among two- and three-element series as follows. It is as easy to learn that a series is a monotonically decreasing one in a two- as in a threeelement series, but it is easier to locate the zero pellet in the shorter series. However, whether shorter series always lead to better anticipation remains to be seen. The usefulness of the rule approach in relation to other findings obtained here is not entirely clear. Why, for example, on Trial 1 of each day, three-element series produced faster running than two-element series is not clear. Nor is it clear in the absence of further specification why some three-element monotonically decreasing series should produce better anticipation than others.

\section{REFERENCES}

Capaldi, E. J. A sequential hypothesis of instrumental learning. In K. W. Spence \& J. T. Spence (Eds.), The psychology of learning and motivation (Vol. 1), New York: Academic Press, 1967.

CAPAldi, E. J. Partial reward either following or preceding consistent reward: A case of reinforcement level. Journal of Experimental Psychology, 1974, 102, 954-962.

CAPAldi, E. J. Latent discrimination learning under a regular schedule of partial reinforcement. Animal Learning \& Behavior, 1979, 7, 63-68.

Capaldi, E. J., \& Molina, P. Element discriminability as a determinant of serial-pattern learning. Animal Learning \& Behavior, in press.

Crowder, R. G. Principles of learning and memory. Hillsdale, NJ: Erlbaum, 1976.

Hulse, S. H. Cognitive structure and serial pattern learning by animals. In S. H. Hulse (Ed.), Cognitive processes in animal behavior. Hillsdale, NJ: Erlbaum, 1978.

Hulse, S. H., \& Dorsky, N. P. Structural complexity as a determinant of serial pattern learning. Learning and Motivation, $1977,8,488-506$.

Restle, F., \& Brown, E. Organization of serial pattern learning. In Gordon Bower (Ed.), The psychology of learning and motivation (Vol. 4). New York: Academic Press, 1970.

Slamecka, N. J. A case for response produced cues in serial learning. Journal of Experimental Psychology: Human Learning \& Motivation, 1977, 3, 222-232.

WiCkelgren, W. A. Context-sensitive coding, associative memory, and serial order in (speech) behavior. Psychological Review, $1969,76,1-15$.

(Received for publication March 26, 1979.) 